Original Article

\title{
To Determine the Diagnostic Accuracy of Curved Multiplanar Reformat of Multidetector CT Scan in Visualizing Zone of Transition in Patients with Intestinal Obstruction
}

\author{
Adnan Iqbal, Sikander Shahzad, Pashmina Gul, Bisham Kumar
}

\section{ABSTRACT}

OBJECTIVE: To determine the diagnostic accuracy of curved Multiplanar reformat of multidetector CT scan in visualizing zone of transition in patients with intestinal obstruction.

SETTING: Radiology and Emergency Department of Dr. Ziauddin University Hospital, Clifton Campus, Karachi.

DESIGN: Cross-sectional descriptive study.

METHODOLOGY: This prospective non funded study was done in Ziauddin University Hospital Clifton campus from January 2014 to July 2015. Patients' curved MPR images results were compared with operative findings taken as gold standard.

RESULT: In this study pre-surgical CT correlation was done in sixty patients with intestinal obstruction, twenty eight were male and thirty two females. Surgical findings suggest that forty four patients were correctly diagnosed on CT scan, giving specificity $85.7 \%$ sensitivity of $95.6 \%$ and diagnostic accuracy of $93.3 \%$.

CONCLUSION: The curved Multiplanar reformat of multidetector CT scan is excellent diagnostic tool and plays a vital role in characterizing and locating area of transition in patients with intestinal obstruction and for relevant treatment.

KEYWORDS: Obstruction, Multiplanar, Transition.

This article may be cited as: Iqbal A, Shahzad S, Gul P, Kumar B. To Determine the Diagnostic Accuracy of Curved Multiplanar Reformat of Multidetector CT Scan in Visualizing Zone of Transition in Patients with Intestinal Obstruction. J Liaquat Uni Med Health Sci. 2017;16(01):37-40. doi: 10.22442/jlumhs. 171610503

\section{INTRODUCTION}

Bowel obstruction is frequently seen clinical problem and in suspected cases of acute abdomen morbidity across the world is approximately $20 \%{ }^{1,2}$. In patients with crampy abdominal pain, distension, vomiting and altered bowel sounds suggesting intestinal obstruction, multiple radiological imaging are done to localize the cause of obstruction including conventional radiography, contrast studies like barium and CT scan. Plain radiography has specificity of $57 \%$ and sensitivity of $69 \%{ }^{1}$ with accuracy between $46-80$ $\%^{3}$. In acute abdomen, first modality for confirmation is plain radiography because of cost effectiveness and easy availability ${ }^{4}$. Clinical finding of small bowel obstruction are when associated with leukocytosis and elevated amylase, suggestive of additional complication which should be assessed by use of cross sectional imaging, beyond helical CT. Multidetector CT (MDCT) is superior in evaluation, proper localization and in detecting complication ${ }^{5-10}$. In recent years role of MDCT has shown promising results in facilitation and demonstration of transitional zone in cases of small bowel obstruction ${ }^{11}$. Helical CT plays a pivotal role in detecting visceral status. Sagittal and coronal reformatted images in MDCT shows promising results in localization of disease and surgical planning in comparison with axial image alone which shows some limitation in thin patients due to less intraperitoneal fat interface between bowel loops ${ }^{12-13}$. It demarcate area of narrowing in cases with obstruction and also access complications like compromised blood and possible leakage. Hodel $\mathrm{J}$ et al study showed increase accuracy and confidence interval of MDCT in cases of bowel obstruction ${ }^{14}$.

This study conducted to determine the diagnostic accuracy of curved MPR in the diagnosis of intestinal obstruction patients by taking surgical findings as gold standard. This was analyzed prospectively and conclusion were derived about the future utility curved MPR in evaluation of intestinal obstruction.

\section{SUBJECTS AND METHODS}

This cross sectional descriptive study was conducted at Radiology and Emergency Department of 
Dr. Ziauddin University Hospital, Clifton Campus, Karachi. During period of study, sixty patients with clinical suspicion of intestinal obstruction were preoperatively screened by CT scan. The Curved MPR results were compared with surgical finding taken as gold standard reference. There were thirty two female and twenty eight male patients. The age range of the patients was between 15 years to 80 years (mean age $49.7 \pm S D$ 18.7years). The sample technique used was purposive non probability type.

After taking informed consent from all patients, clinical history taken including history of previous surgery. Patients who did not consented were excluded from study.

Images of CT scan of abdomen obtained on multidetector ASTEION 16 (Toshiba JAPAN) with section of $5 \mathrm{~mm}$ from level of D12 vertebral body to pubic symphysis. All the patients underwent multidetector CT with oral and intravenous contrast portal venous phase before surgical exploration with protocol of collimation of $2 \mathrm{~mm}$, pitch $1.35,120 \mathrm{kvp}$, $250 \mathrm{mAs}$ and medium FOV. Multiplanar reformats with curved MPR were acquired in each patient besides routine axial image. The CT findings were compared with surgical findings in determining accuracy of curved MPR in detecting zone of transition between distended and collapsed bowel loops. The added curved MPR images showed high accuracy in diagnosis of bowel obstruction with unremarkable advantage over conventional axial images. Images were reviewed by two radiologists. All patients were followed, and operative findings of all patients who undergo subsequent surgery were collected and compared with computed tomography findings. All concerned data were collected and Statistical analysis was performed with SPSS software. The site and level of obstruction were evaluated. Negative predictive values, positive predictive value, specificity and sensitivity values of Curved MPR were calculated and compared with operative results taken as gold standard, $p$ value of equal to or less than 0.05 was considered level of significance.

\section{RESULTS}

This study was conducted for a period of one and half year in which pre surgical CT was done on sixty patients, thirty two female and twenty eight males with age between fifteen to eighty years. Forty four patients were correctly diagnosed pre-surgically on $\mathrm{CT}$ in this study as shown in Table No. 1 with sensitivity of 95.6 $\%$, twelve patients were truly diagnosed negative with specificity of $85.7 \%$ and accuracy of $93.3 \%$ detected on curved MPR in localizing zone of transition in cases of intestinal obstruction. CT scan has positive predictive value of $86.9 \%$ and negative predictive value of $85.7 \%$. Chart No. 2 delineate causes of obstruction seen based on operative findings; adhesions $(43.2 \%)$, hernias $(18.1 \%)$, tumor $(4.5 \%)$, Tuberculosis $(9 \%)$, foreign bodies $(4.5 \%)$ and ischemia in $(40.9 \%)$ on CT scan.

Figure No. 1: (1D) imply a segment of collapsed small bowel (white arrow), but, the confidence is very low. Coronal enhanced MDCT images (1E) fail to display the transition zone of obstruction. CPR images (1F) reveal clearly the transition zone (white arrow) and the location of the obstruct site is more reliable.

\section{CHART I: GENDER DISTRIBUTION}

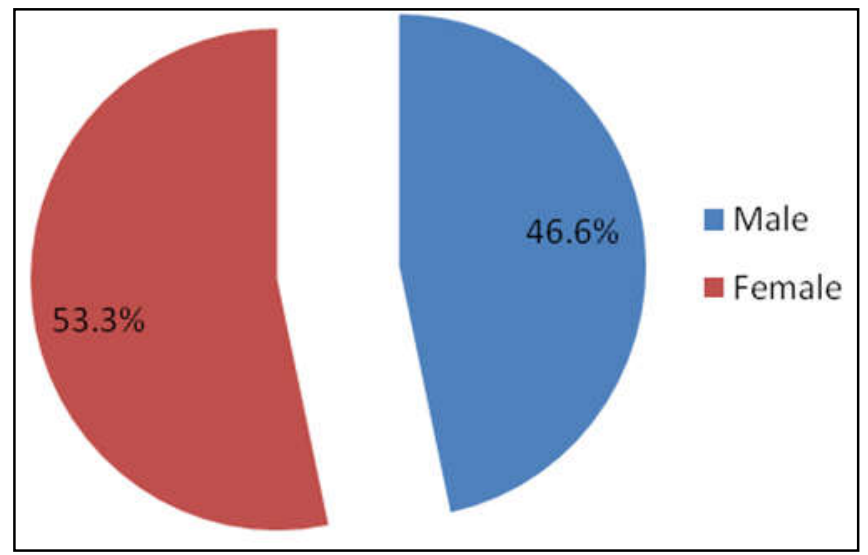

\section{CHART II: OBSTRUCTION CAUSES}

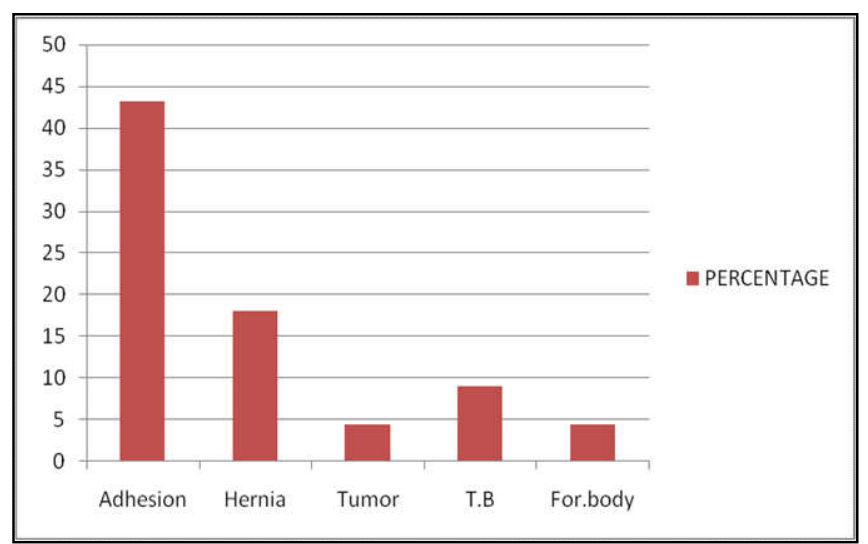

TABLE I: SUMMARY

\begin{tabular}{|l|c|c|c|}
\hline & $\begin{array}{c}\text { Surgical } \\
\text { Finding } \\
\text { +VE }\end{array}$ & $\begin{array}{c}\text { Surgical } \\
\text { Finding } \\
\text { +VE }\end{array}$ & Total \\
\hline CT DAIGNOSIS+VE & 44 & 02 & 46 \\
\hline CT DAIGNOSIS- VE & 02 & 12 & 14 \\
\hline TOTAL & 46 & 14 & 60 \\
\hline
\end{tabular}




\section{TABLE II: RESULTS}

\begin{tabular}{|l|c|c|}
\hline \multicolumn{1}{|c|}{ Statistical Measure } & Numbers & \% \\
\hline Sensitivity & $44 / 60$ & $95.6 \%$ \\
\hline Specificity & $12 / 14$ & $85.7 \%$ \\
\hline Accuracy & $56 / 60$ & $93.3 \%$ \\
\hline PPV & $40 / 46$ & $86.9 \%$ \\
\hline NPV & $12 / 14$ & $85.7 \%$ \\
\hline
\end{tabular}

FIGURE NO. I:

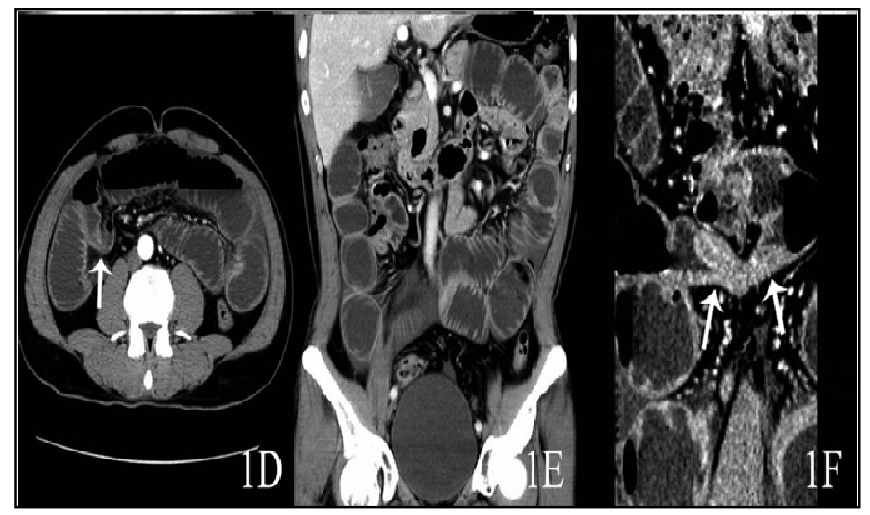

\section{DISCUSSION}

Recent advances have been made in CT technology since its introduction thirty years ago. The arrival of MDCT into clinical practice has been a major development and best diagnostic tool in delineating zone of transition ${ }^{2}$. It is possible to obtain z-axis resolution with high quality two-and three- dimensional reformations. Imaging extent has been dramatically increased and examination times significantly reduced, which allows imaging of organs in well defined perfusion phases ${ }^{16}$. Reduced screening time shows improvement in mesentery and gut evaluation 17,18. There are multiple causes of bowel obstruction that include intrinsic, extrinsic and intraluminal. The intraluminal causes include tuberculosis, intussusception adenocarcinoma, Crohn disease, extrinsic causes like close loop obstruction, hernia, adhesions, tumors appendicitis, diverticulitis and intraluminal causes include foreign bodies like gossypiboma, bezoars and cholelithiasis $^{16}$. In some cases of adhesion contrast hold up seen so followed up to twenty four hour for its passage if not then surgery recommended ${ }^{19}$. In some cases of bowel volvulus MPR localize cause and shows twisted segment ${ }^{20}$. In some cases close loop obstruction seen as $U$ shaped, C shaped or coffee bean configuration at twisting segment ${ }^{21}$. Surgical exploration performed in these patients, adhesion followed by hernia is most prevalent causes of bowel obstruction.

In the previous studies there is limited role of multiplanar reconstruction images. Coronal reformatted images shows significant increase in confidence level and inter-observer agreement in detection of bowel obstruction however does not reflect the role of MPR in localization zone of transition as proposed by Jaffrey RB study. Hodel J showed accuracy of $93 \%$ by using MPR in locating transition zone ${ }^{14}$. One prospective study shows sensitivity of ischemia detection is approximately $15 \%{ }^{23}$. More recent articles describe various CT signs and findings in small bowel obstruction to be managed conservatively or surgically ${ }^{24-28}$. In our study we proved the additional diagnostic value of this technique and showed accuracy of $93.8 \%$ by two radiologist's findings results.

\section{CONCLUSION}

Multiplanar reformat technique is excellent diagnostic tool in detecting zone of transition in cases of intestinal obstruction and for proper treatment.

\section{REFERENCES}

1. Foster NM, McGory ML, Zingmond DS, Ko CY. "Small bowel obstruction: a population-based appraisal. J Am Coll Surg. 2006;203(2):170-6.

2. Jeffrey RB. Small bowel obstruction. In: Federle $M P$, Jeffrey RB, Woodward PJ, Borhani AA, eds. Diagnostic imaging: abdomen, 2nd ed. Salt Lake City, Utah: Amirsys, 2010; 44-7.

3. Cahir JG, Coutney AH, Courtney HM. Multislice CT of the abdomen. BJR 2004;77(Suppl 1):S64S73.

4. Thompson WM, Kilani RK, Smith BB, et al. Accuracy of abdominal radiography in acute small -bowel obstruction: does reviewer experience matter? AJR 2007;188:757;[web]W233-W238.

5. Silva AC, Pimenta M, Guimarães LS. Small bowel obstruction: what to look for. Radiographics 2009;29(2):423-39.

6. Atri M, McGregor C, Mclnnes M, et al. Multidetector helical CT in the evaluation of acute small bowel obstruction: comparison of non-enhanced (no oral, rectal or IV contrast) and IV enhanced CT. Eur J Radiol 2009;71(1):135-140.

7. Goussous N, Eiken PW, Bannon MP, Zielinski MD. Enhancement of a small bowel obstruction model using the gastrografin $₫$ challenge test. J Gastrointest Surg 2013;17(1):110-116.

8. Catena F, Di Saverio S, Kelly MD, et al. Bologna guidelines for diagnosis and management of adhesive small bowel obstruction (ASBO): 2010 evidence-based guidelines of the World Society of Emergency Surgery. World J Emerg Surg 2011;6:5.

9. Wiesner W, Mortele K. Small bowel ischemia 
caused by strangulation in complicated small bowel obstruction: CT findings in 20cases with histopathological correlation. JBR-BTR 2011;94 (6):309-14.

10. Santillan CS. Computed tomography of small bowel obstruction. Radiol Clin North Am 2013;51 (1):17-27.

11. Desser TS, Gross M. 2008. Multidetector row computed tomography of small bowel obstruction. Semin Ultrasound CT MR. 29:308-21.

12. Shah ZK, Uppot RN, Wargo JA, Hahn PF, Sahani DV. Small bowel obstruction: The value of coronal reformatted images from 16-multidetector computed tomography--aclinicoradiological perspective. J Comput Assist Tomogr. 2008;32:23-31.

13. Frager D, Medwid SW, Baer JW, Mollinelli B, Friedman M. CT of small-bowel obstruction: value in establishing the diagnosis and determining the degree and cause. AJR Am J Roentgenol 1994;162(1):37-41.

14. Taourel PG, Fabre JM, Pradel JA, Seneterre EJ, Megibow AJ, Bruel JM. Value of CT in the diagnosis and management of patients with suspected acute small-bowel obstruction. AJR Am J Roentgenol 1995;165(5):1187-92

15. J. Hodel, M. Zins, L. Desmottes et al. Location of the transition zone in CT of small-bowel obstruction: added value of multiplanar reformations. Abdominal Imaging.2009; 34(1): 35-41

16. Mahmoud M, Al-Hawary, Kaza RK, Platt JF. MDCT and 3D imaging of the small bowel and mesentery. Appl Radiol 2011;40:11

17. Johnson PT, Horton KM, Fishman EK. Nonvascular mesenteric disease: Utility of multidetector CT with 3D volume rendering. Radiographics 2009;29:721-40

18. Barnett RE, Younga J, Harris B, et al. Accuracy of computed tomography in small bowel obstruction. Am Surg 2013;79(6): 641-3.

19. Vandendries $C$, Jullès MC, Boulay-Coletta I, Loriau J, Zins M. Diagnosis of colonic volvulus: Findings on multidetector CT with threedimensional reconstructions. Br J Radiol2010;83
(995):983-90.

20. Hayakawa K, Tanikake M, Yoshida S, Yamamoto A, Yamamoto E, Morimoto T. CT findings of small bowel strangulation: the importance of contrast enhancement. Emerg Radiol 2013;20(1):3-9.

21. Maglinte DD, Heitkamp DE, Howard TJ, Kelvin FM, Lappas JC. Current concepts in imaging of small bowel obstruction. Radiol Clin North Am. 2003;41(2):263-83

22. Sheedy SP, Earnest $F$ 4th, Fletcher JG, Fidler JL, Hoskin TL. CT of small-bowel ischemia associated with obstruction in emergency department patients: diagnostic performance evaluation. Radiology 2006;241(3):729-36.

23. Rocha FG, Theman TA, Matros E, Ledbetter SM, Zinner MJ, Ferzoco SJ. Non operative management of patients with a diagnosis of high-grade small bowel obstruction by computed tomography. Arch Surg 2009;144(11):1000-4

24. Idris $M$, Kashif $N$, Idris $S$, Memon WA, Tanveer UH, Haider Z. Accuracy of 64-slicemultidetector computed tomography scan in detection of the point of transition of small bowel obstruction. Jpn J Radiol 2012;30(3):235-41

25. Zielinski MD, Eiken PW, Bannon MP, et al. Small bowel obstruction-Who needs an operation? a multivariate prediction model. World J Surg 2010;34(5):910-19.

26. Kato K, Mizunuma K, Sugiyama M, et al. Interobserver agreement on the diagnosis of bowel ischemia: assessment using dynamic computed tomography of small bowel obstruction.Jpn J Radiol 2010;28(10):727-32.

27. Jang KM, Min K, Kim MJ, et al. Diagnostic performance of CT in the detection of intestinal ischemia associated with small-bowel obstruction using maximal attenuation of region of interest. AJR Am J Roentgenol. 2010;194(4):957-63.

28. Deshmukh SD, Shin DS, Willmann JK, Rosenberg $J$, Shin L, Jeffrey RB. Non-emergency small bowel obstruction: assessment of CT findings that predict need for surgery. Eur Radiol. 2011;21(5):982-6.

\section{AUTHOR AFFILIATION:}

Dr. Adnan Iqbal (Corresponding Author) Resident, Department of Emergency Medicine Ziauddin University \& Hospital, Karachi, Sindh-Pakistan. E-mail: adnan.shaikh82@gmail.com

Dr. Sikander Shahzad

Resident, Department of Radiology Ziauddin University \& Hospital, Karachi, Sindh-Pakistan.

\section{Dr. Pashmina Gul}

Radiologist

Ziauddin University \& Hospital, Karachi, Sindh-Pakistan.

Dr. Beesham Kumar

Radiologist

Ziauddin University \& Hospital, Karachi, Sindh-Pakistan. 